\title{
HAUSDORFF DIMENSION OF SETS ARISING IN DIOPHANTINE APPROXIMATION
}

\author{
TAKANORI HINOKUMA AND HIROO SHIGA
}

\begin{abstract}
Let $g(q)$ be a nonnegative function on the set of positive integers. We studied the Hausdorff dimension of a set

$$
E_{\boldsymbol{g}}=\left\{x \in[0,1]:\left|x-\frac{p}{q}\right|<g(q) \text { for infinitely many } \frac{p}{q}\right\} .
$$

We prove a generalization of a result of I. Borosh and A.S. Fraenkel.
\end{abstract}

\section{Introduction}

It was shown by Jarnik and Besicovitch that the Hausdorff dimension, denoted by $\operatorname{dim}_{H}$, of the set of real numbers for which there exist infinitely many rationals $p / q$ satisfying

$$
\left|x-\frac{p}{q}\right|<\frac{1}{q^{\beta}}
$$

is $\min \{2 / \beta, 1\}$. In 1972 Borosh and Fraenkel extended the above result in the following way. Let $\mathcal{L}$ be a subset of positive integers having infinitely many elements and set

$$
E_{\mathcal{L}}=\left\{x \in[0,1]:\left|x-\frac{p}{q}\right|<\frac{1}{q^{\beta}} \text { for infinitely many } \frac{p}{q} \text { with } q \in \mathcal{L}\right\} .
$$

THEOREM B-F ([1]). Let $\nu_{0}$ be a real number satisfying the following two conditions :

(i) $\sum_{q \in \mathcal{L}} q^{-\nu_{0}}$ is divergent,

(ii) $\sum_{q \in \mathcal{L}} q^{-\nu_{0}-\varepsilon}$ is convergent for every $\varepsilon>0$.

Then $\operatorname{dim}_{H} E_{\mathcal{L}}=\min \left\{\left(1+\nu_{0}\right) / \beta, 1\right\}$.

The purpose of the present paper is to study the Hausdorff dimension of a set

1991 Mathematics Subject Classification. 11J83, 28A78.

Received October 24, 1995 ; revised June 5, 1996. 


$$
E_{g}=\left\{x \in[0,1]:\left|x-\frac{p}{q}\right|<g(q) \text { for infinitely many } \frac{p}{q}\right\},
$$

where $g$ is a nonnegative function on the set of positive integers. We set

$$
C_{\alpha}(N)=\text { the cardinality of the set }\left\{q \leqq N: g(q) \geqq \frac{1}{q^{\alpha}}\right\}
$$

and

$$
\gamma(\alpha)=\sup \left\{\gamma: \varlimsup_{N \rightarrow \infty} \frac{C_{\alpha}(N)}{N^{r}}>0\right\} .
$$

Then we shall prove the following result:

THEOREM 1.1.

$$
\operatorname{dim}_{H} E_{g}=\min \left\{\sup _{\alpha \geq 1} \delta(\alpha), 1\right\}
$$

where

$$
\delta(\alpha)=\left\{\begin{array}{cl}
\frac{1+\gamma(\alpha)}{\alpha} & \text { if } \lim _{N \rightarrow \infty} C_{\alpha}(N)=\infty \\
0 & \text { otherwise. }
\end{array}\right.
$$

Let $f(q)$ be a function on the set positive integers with the values 0 or 1. We consider the case

$$
g(q)=\frac{f(q)}{q^{\alpha_{0}}} .
$$

In this case, $C_{\alpha}(N)$ is equal to the cardinality of the set $\{q \leqq N: f(q)=1\}$ if $\alpha \geqq \alpha_{0}$ and $C_{\alpha}(N)=0$ if $\alpha<\alpha_{0}$. Then $\delta(\alpha)=\left(1+\gamma\left(\alpha_{0}\right)\right) / \alpha$ if $\alpha \geqq \alpha_{0}$ and $\delta(\alpha)=0$ if $\alpha<\alpha_{0}$. In this situation, we denote $E_{f / q} \alpha_{0}$ by $E_{f}$ for simplicity and set $\gamma_{0}=$ $\gamma\left(\alpha_{0}\right)$. Then Theorem 1.1 reduces to the following

PROPOSITION 1.2.

$$
\operatorname{dim}_{H} E_{f}=\min \left\{\frac{1+\gamma_{0}}{\alpha_{0}}, 1\right\}
$$

Set $f(q)=1$ if $q \in \mathcal{L}$ and $f(q)=0$ if $q \notin \mathcal{L}$. Then Theorem B-F can be obtained from Proposition 1.2 by proving

$$
\nu_{0}=\gamma_{0}
$$

We prove Proposition 1.2 and $\nu_{0}=\gamma_{0}$ in $\S 2$. A proof of Theorem 1.1 is given in $\S 3$. In $\S 4$, some examples are given.

\section{Proof of Proposition 1.2}

In this section, we give the proof of the Proposition 1.2. We first show 
the inequality $\operatorname{dim}_{H}\left(E_{f}\right) \leqq\left(1+\gamma_{0}\right) / \alpha_{0}$. For each positive integer $q$, we set

$$
F_{q}=\left\{x \in[0,1]:\left|x-\frac{p}{q}\right|<\frac{f(q)}{q^{\alpha_{0}}} \text { for some integer } p\right\} .
$$

Then $F_{q}$ consists of $q-1$ intervals of length $2 f(q) / q^{\alpha_{0}}$ and two end intervals of length $f(q) / q^{\alpha_{0}}$. Clearly, $E_{f} \subset \bigcup_{q=k}^{\infty} F_{q}$ for each positive integer $k$, so taking the intervals of $F_{q}$ for $q \geqq k$ as a cover of $E_{f}$ gives that

$$
\mathscr{H}_{\delta}^{s}\left(E_{f}\right) \leqq \sum_{q=k}^{\infty}(q+1)\left(\frac{2 f(q)}{q^{\alpha_{0}}}\right)^{s}
$$

If $2 / k^{\alpha_{0}} \leqq \delta$, where $\mathscr{H}_{\delta}^{s}(E)$ is the infimum of $\sum_{\imath=1}^{\infty}\left|U_{\imath}\right|^{s}$ over all countable $\delta$-covers $\left\{U_{i}\right\}$ of $E$. The right hand of the above inequality is smaller than

$$
\sum_{q=k}^{\infty} 2 q\left(\frac{2 f(q)}{q^{\alpha_{0}}}\right)^{s}=2^{s+1} \sum_{q=k}^{\infty} \frac{f(q)}{q^{s \alpha_{0}-1}} .
$$

Hence if the series $\sum_{q=k}^{\infty} f(q) / q^{s \alpha_{0}-1}$ converges, then the Hausdorff $s$-dimensional measure $\mathscr{H}^{s}\left(E_{f}\right)=\lim _{\delta \rightarrow 0} \mathscr{H}_{\delta}^{s}\left(E_{f}\right)=0$.

Fix a positive real number $\gamma$ with $\gamma>\gamma_{0}$. Let $C_{f}(N)$ be the cardinality of the set $\{q: q \leqq N$ and $f(q)=1\}$. Note that $C_{f}(N)=C_{\alpha_{0}}(N)$. Then, for $\lim _{N \rightarrow \infty} C_{f}\left(N^{1 / r}\right) / N=\lim _{N \rightarrow \infty} C_{f}(N) / N^{r}=0$, there exists an integer $N_{0}$ such that if $N \geqq N_{0}$ then $C_{f}\left(N^{1 / r}\right) \leqq N$. We define $M_{k}$ by $C_{f}\left(M_{k}\right)=k N_{0}(k=1, \cdots)$. Since $C_{f}\left(\left(k N_{0}\right)^{1 / r}\right)<k N_{0}=C_{f}\left(M_{k}\right)$, we have

$$
\left(k N_{0}\right)^{1 / r}<M_{k} .
$$

Set

$$
\mathscr{M}_{k}=\left\{q: f(q)=1 \text { and } M_{k-1}<q \leqq M_{k}\right\}
$$

then $\# \mathscr{M}_{k}=N_{0}$ from the definition of $M_{k}$. Now we have

$$
\begin{aligned}
\sum_{q=1}^{\infty} \frac{f(q)}{q^{s \alpha_{0}-1}}=\sum_{k=0}^{\infty} \sum_{q \in \mathcal{S M}_{k+1}} \frac{1}{q^{s \alpha_{0}-1}} & <\sum_{q \in \mathscr{M}_{1}} \frac{1}{q^{\beta}}+\sum_{k=1}^{\infty} \frac{N_{0}}{\left(\left(k N_{0}\right)^{1 / r}\right)^{s \alpha_{0}-1}} \\
& <N_{0}+\frac{1}{N_{0}^{\left(s \alpha_{0}-1\right) / \gamma}} \sum_{k=1}^{\infty} \frac{1}{k^{\left(s \alpha_{0}-1\right) / \gamma}} .
\end{aligned}
$$

If $s>(1+\gamma) / \alpha_{0}$, then $\sum_{k=1}^{\infty} 1 / k^{\left(s \alpha_{0}-1\right) / \gamma}$ is convergent. Hence if $s>(1+\gamma) / \alpha_{0}$ we have $\mathcal{H}^{s}\left(E_{f}\right)=0$ and therefore $\operatorname{dim}_{H}\left(E_{f}\right) \leqq(1+\gamma) / \alpha_{0}$ for any $\gamma>\gamma_{0}$. This implies that $\operatorname{dim}_{H}\left(E_{f}\right) \leqq\left(1+\gamma_{0}\right) / \alpha_{0}$.

We need some lemmas to prove converse inequality. Let $C_{f}(N, M)$ be the cardinality of the set $\{q: N \leqq q \leqq M$ and $f(q)=1\}$. We set

$$
\gamma_{1}=\sup \left\{\gamma: \varlimsup_{N \rightarrow \infty} \frac{C_{f}(N, 2 N)}{N^{r}}>0\right\} .
$$

LEMMA 2.1 .

$$
\gamma_{1}=\gamma_{0}
$$


Proof. It is clear that $0 \leqq \gamma_{1} \leqq \gamma_{0}$. Hence it is sufficient to show that $\gamma_{1} \geqq \gamma_{0}$. We can assume that $\gamma_{0}>0$. Let $\varepsilon$ be a positive number with $\gamma_{0}-\varepsilon>0$ and $\left\{n_{j}\right\}$ be a sequence of positive integers such that

$$
\frac{C_{f}\left(n_{j}\right)}{\left(\log _{2} n_{j}\right) n_{j}^{\gamma_{0}}} \cdot n_{j}^{\varepsilon} \rightarrow \infty \quad(\jmath \rightarrow \infty) .
$$

It is possible to choose such a sequence as above by definition of $\gamma_{0}$. We divide the interval $[1, n$,$] into k$-small intervals

$$
\left[1, \frac{n_{\jmath}}{2^{k}}\right),\left[\frac{n_{\jmath}}{2^{k}}, \frac{n_{\jmath}}{2^{k-1}}\right), \cdots,\left[\frac{n_{\jmath}}{2}, n_{\jmath}\right],
$$

where $k$ is the greatest integer satisfying $k<\log _{2} n_{\jmath}$. Let $m$ be the number such that $C_{f}\left(n_{j} / 2^{m+1}, n_{j} / 2^{m}\right)$ is the greatest among $C_{f}\left(n_{j} / 2^{l+1}, n_{j} / 2^{l}\right), l=0,1, \cdots, k$.

Let $M_{j}=n_{j} / 2^{m}$. Then $M_{j} \rightarrow \infty$ if $j \rightarrow \infty$. Because if $M_{j}<K$ for some constant $K$, then we have

$$
\begin{aligned}
C_{f}(K) & \geqq C_{f}\left(M_{\jmath}\right) \\
& \geqq \frac{C_{f}\left(n_{j}\right)}{\log _{2} n_{\jmath}} \\
& =\frac{C_{f}\left(n_{j}\right)}{\left(\log _{2} n_{\jmath}\right) n_{\jmath}^{\gamma_{0}}} \cdot n_{j}^{\varepsilon} \cdot n_{j}^{\gamma_{0}-\varepsilon} \rightarrow \infty \quad(j \rightarrow \infty) .
\end{aligned}
$$

This is a contradiction.

Now for $\gamma_{0}-\varepsilon>0$,

$$
\frac{C_{f}\left(n_{j}\right)}{n_{j}^{\gamma_{0}^{-\varepsilon}}} \leqq \frac{\left(\log _{2} n_{j}\right) C_{f}\left(M_{j} / 2, M_{j}\right)}{M_{j}^{\gamma^{-\varepsilon}}} .
$$

Since $C_{f}\left(n_{j}\right) /\left(\log _{2} n_{j}\right) n_{j}^{\gamma_{0}-3} \rightarrow \infty(j \rightarrow \infty)$ we have

$$
\frac{C_{f}\left(M_{j} / 2, M_{j}\right)}{M_{j^{-\varepsilon}}^{r_{0}}} \rightarrow \infty \quad(j \rightarrow \infty)
$$

Hence $\gamma_{1} \geqq \gamma_{0}-\varepsilon$. Since $\varepsilon$ can be taken arbitrary small, it follows that $\gamma_{1} \geqq \gamma_{0}$.

Let $P(N)=N^{r_{0}} / C_{f}(N, 2 N)$. Then by Lemma 2.1

$$
\lim _{N \rightarrow \infty} N^{\varepsilon} P(N)=+\infty \text { and } \lim _{N \rightarrow \infty} N^{-\varepsilon} P(N)=0
$$

for any $\varepsilon>0$. So, for each $\varepsilon>0$, we choose a sequence of positive integers $\left\{N_{j}\right\}$ such that $N_{j}>2 N_{\jmath-1}$ and

$$
\lim _{j \rightarrow \infty} N_{j}^{\varepsilon} P\left(N_{j}\right)=+\infty \text { and } \lim _{j \rightarrow \infty} N_{j}^{-\varepsilon} P\left(N_{j}\right)=0 .
$$

First we consider the case $\alpha_{0}>1+\gamma_{0}$. Set $L_{\jmath}=\left\{N_{\jmath} \leqq q \leqq 2 N_{\jmath}: f(q)=1\right\}$ and $l_{\jmath}=\# L_{\jmath}=C_{f}\left(N_{\jmath}, 2 N_{\jmath}\right)$. Then $l_{j} P\left(N_{j}\right) / N_{\jmath}^{\gamma_{0}}=1$. Let $L_{\jmath}=\left\{q_{1}, \cdots, q_{l_{j}}\right\}$ with $N_{\jmath} \leqq$ $q_{1}<q_{2}<\cdots<q_{l_{j}} \leqq 2 N_{\jmath}$. Let $\tilde{G}_{q_{k}}$ be the set of reduced fractions $p / q_{k}$ in the 
interval $[0,1]$ whose numerators $p$ are prime numbers. Let $G_{q_{1}}=\tilde{G}_{q_{1}}$ and let $G_{q_{2}} \subset \tilde{G}_{q_{2}}$ be the set of reduced fractions $t_{2} / q_{2}$ in the interval $[0,1]$, whose numerators are prime numbers, satisfying

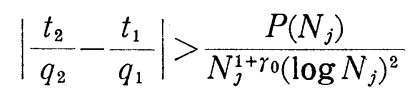

for any element $t_{1} / q_{1}$ of $G_{q_{1}}$.

LEMMA 2.2 .

$$
\#\left(\tilde{G}_{q_{2}}-G_{q_{2}}\right) \leqq \frac{8 N_{j}^{1-\gamma_{0} P\left(N_{\jmath}\right)}}{\left(\log N_{j}\right)^{2}} .
$$

Proof. If $t_{2} / q_{2}$ satisfies the inequality

$$
\left|t_{2} q_{1}-t_{1} q_{2}\right|>\frac{4 N_{j}^{1-\gamma_{0}} P\left(N_{j}\right)}{\left(\log N_{j}\right)^{2}}
$$

for any $t_{1} / q_{1} \in G_{q_{1}}$, it satisfies (1) for any $t_{1} / q_{1} \in G_{q_{1}}$. So we count up the number of fractions $t_{2} / q_{2}$ which satisfies

$$
\left|t_{2} q_{1}-t_{1} q_{2}\right| \leqq \frac{4 N_{\jmath}^{1-\gamma_{0}} P\left(N_{j}\right)}{\left(\log N_{\jmath}\right)^{2}}
$$

for some $t_{1} / q_{1} \in G_{q_{1}}$. Since the number of solutions $\left(t_{1}, t_{2}\right)$ of the equation

$$
\left|t_{2} q_{1}-t_{1} q_{2}\right|=k \quad(k \text { is a positive integer })
$$

in the range $0 \leqq t_{1} \leqq q_{1}, 0 \leqq t_{2} \leqq q_{2}$ is at most two, the number of reduced fractions $t_{2} / q_{2}$ which satisfies (2) for some $t_{1} / q_{1} \in G_{q_{1}}$ is at most $8 N_{j}^{1-r_{0}} P\left(N_{j}\right) /\left(\log N_{j}\right)^{2}$. Hence we have the lemma.

Now we inductively define $G_{q_{k}} \subset \tilde{G}_{q_{k}}$. Let $G_{q_{k}}$ be a set of reduced fractions $t_{k} / q_{k}$ with prime numerators which satisfies

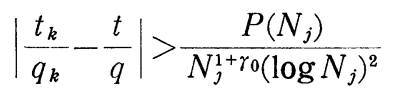

for all $t / q \in \bigcup_{i=1}^{k-1} G_{q_{i}}$. Then by the similar argument as the proof of Lemma 2.2, we have

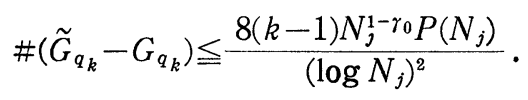

Set $H_{j}=\bigcup_{k=1}^{l_{3}} G_{q_{k}}$ and $\tilde{H}_{j}=\bigcup_{k=1}^{l_{3}} \tilde{G}_{q_{k}}$. Then we get

LEMMA 2.3.

$$
\#\left(\widetilde{H}_{j}-H_{j}\right)<\frac{4 l_{j} N_{j}}{\left(\log N_{j}\right)^{2}}
$$


Proof. By the preceeding discussion, we have

$$
\#\left(\tilde{H}_{j}-H_{j}\right)=\sum_{k=1}^{l_{j}} \#\left(\tilde{G}_{q_{k}}-G_{q_{k}}\right) \leqq \sum_{k=1}^{l_{j}} \frac{8(k-1) N_{j}^{1-\gamma_{0}} P\left(N_{j}\right)}{\left(\log N_{j}\right)^{2}} .
$$

Therefore we have

$$
\begin{aligned}
\#\left(\tilde{H}_{j}-H_{j}\right) \leqq & \frac{4 l_{j}\left(l_{j}-1\right) N_{j}^{1-\gamma_{0}} P\left(N_{\jmath}\right)}{\left(\log N_{j}\right)^{2}} \\
& <\frac{4 l_{j} N_{\jmath}}{\left(\log N_{j}\right)^{2}} \cdot \frac{l_{j} P\left(N_{j}\right)}{N_{j}^{\gamma_{0}}} \\
& =\frac{4 l_{j} N_{j}}{\left(\log N_{j}\right)^{2}},
\end{aligned}
$$

since $l_{j} P\left(N_{j}\right) / N_{j}^{\gamma_{0}}=1$.

Now we complete the proof of Proposition 1.2. For any two different elements $p_{1} / r_{1}$ and $p_{2} / r_{2}$ of $H_{\jmath}$, we have

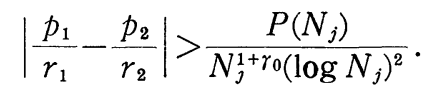

Set

and

$$
I_{p / q}\left\{x \in[0,1]:\left|x-\frac{p}{q}\right|<\frac{f(q)}{q^{\alpha_{0}}}\right\}
$$

$$
K_{\jmath}=\bigcup_{p / q \in H_{\jmath}} I_{p / q}
$$

Then the distance between two different intervals $I_{p / q}$ and $I_{p^{\prime} / q^{\prime}}$ is at least

$$
\frac{P\left(N_{j}\right)}{N_{\jmath}^{1+\gamma_{0}}\left(\log N_{j}\right)^{2}}-\frac{2}{N_{j}^{\alpha_{0}}}>\frac{P\left(N_{j}\right)}{2 N_{\jmath}^{1+\gamma_{0}}\left(\log N_{j}\right)^{2}}
$$

for sufficiently large $N_{\jmath}$, since $1+\gamma_{0}<\alpha_{0}$.

Let $E_{0}=[0,1]$ and $E_{3}$ the set of intervals of $K$, which are completely contained in some of $E_{\jmath-1}$. Then the intervals of $E_{\jmath}$ are separated by gaps of at least

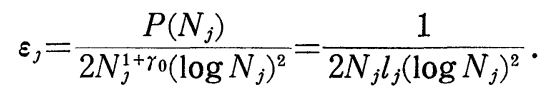

Let $I=[a, b] \subset[0,1]$ be an interval with $|I|>3 / N_{\jmath}$. We count the number of intervals of $K$, in $I$.

LEMMA 2.4. The number of intervals of $K_{3}$ contained in $I$ is at least

$$
\frac{(b-a) N_{j} l_{j}}{16 \log N_{\jmath}},
$$

for sufficiently large $N_{\jmath}$. 
Proof. The number of rationals in $I$ whose denominators are $q_{k}$ and numerators are prime is equal to the number of primes in the interval $\left[a q_{k}, b q_{k}\right]$. By prime number theorem there are at least

$$
\frac{1}{2}\left(\frac{b q_{k}}{\log b q_{k}}-\frac{a q_{k}}{\log a q_{k}}\right)
$$

primes in $\left[a q_{k}, b q_{k}\right]$. Then we have

Then by Lemma 2.3,

$$
\begin{aligned}
\frac{1}{2}\left(\frac{b q_{k}}{\log b q_{k}}-\frac{a q_{k}}{\log a q_{k}}\right) & >\frac{1}{4} \cdot \frac{(b-a) q_{k}}{\log q_{k}}=\frac{1}{4} \cdot \frac{q_{k}|I|}{\log q_{k}} \\
& >\frac{1}{8} \cdot \frac{N_{j}|I|}{\log N_{\jmath}} .
\end{aligned}
$$

$$
\begin{aligned}
\#\left(I \cap H_{\jmath}\right) & =\#\left(I \cap \tilde{H}_{j}\right)-\#\left(I \cap\left(\tilde{H}_{j}-H_{j}\right)\right) \\
& \geqq \frac{1}{8} \cdot \frac{N_{j} l_{j}|I|}{\log N_{\jmath}}-\frac{4 l_{j} N_{\jmath}}{\left(\log N_{j}\right)^{2}} \\
& =l_{j}\left(\frac{N_{j}|I|}{8 \log N_{\jmath}}-\frac{4 N_{\jmath}}{\left(\log N_{\jmath}\right)^{2}}\right) \\
& >\frac{N_{j} l_{j}|I|}{16 \log N_{\jmath}} .
\end{aligned}
$$

Hence at least $N_{j} l_{j}|I| / 16 \log N_{\jmath}$ intervals of $K_{\jmath}$ are contained in $I$ for sufficiently large $N_{\jmath}$.

We can take the sequence $\left\{N_{j}\right\}$ to satisfy the inequality

$$
\frac{2}{\left(2 N_{\jmath-1}\right)^{\alpha}}>\frac{64}{\log N_{\jmath}} \text {. }
$$

As the length of each interval in $E_{\jmath-1}$ is at least $2 /\left(2 N_{\jmath-1}\right)^{\alpha}$, by Lemma 2.4 , $E_{\jmath-1}$ contains at least

$$
m_{\jmath}=\frac{N_{j} l_{j}\left(2 N_{j-1}\right)^{-\alpha_{0}}}{16 \log N_{j}}=\frac{c N_{j} l_{j} N_{\jmath-1}^{-\alpha_{0}}}{\log N_{\jmath}}
$$

intervals of $K_{\jmath}$, where $c=16^{-1} \cdot 2^{-\alpha_{0}}$ and we set $m_{1}=1$. By choosing a subsequence of $N_{\jmath}$, we can assume that $\log N_{\jmath}>j \log N_{\jmath-1}$. Then clearly $\varepsilon_{\jmath}>\varepsilon_{\jmath+1}$ for sufficiently large $j$. By Example 4.6 (p. 58) in [2], we have

$$
\begin{aligned}
& \operatorname{dim}_{H}\left(\bigcap_{\jmath=1}^{\infty} E_{\jmath}\right) \geqq \varliminf_{\jmath \rightarrow \infty} \frac{\log \left(m_{1} \cdots m_{\jmath-1}\right)}{-\log \left(m_{j} \varepsilon_{\jmath}\right)} \\
= & \lim _{\jmath \rightarrow \infty} \frac{\log \left[c^{\jmath-2} N_{1}^{-\alpha_{0}}\left(N_{2} \cdots N_{\jmath-2}\right)^{1-\alpha_{0}}\left(l_{2} \cdots l_{\jmath-2}\right)\left(\log N_{2} \cdots \log N_{\jmath-2}\right)^{-1} l_{\jmath-1} N_{\jmath-1}\right]}{-\log \left[c N_{\jmath-1}^{\left.-\alpha_{0}\left(\log N_{\jmath-1}\right)^{-3}\right]} .\right.}
\end{aligned}
$$

The numerator can be rewritten as following: 


$$
\begin{aligned}
& (j-2) \log c-\alpha_{0} \log N_{1}+\left(1+\gamma_{0}-\alpha_{0}\right)\left(\log N_{2}+\cdots+\log N_{\jmath-2}\right) \\
& -\left(\log \log N_{2}+\cdots+\log \log N_{\jmath-2}\right) \\
& -\left(\log P\left(N_{2}\right)+\cdots+\log P\left(N_{\jmath-2}\right)\right)+\left(1+\gamma_{0}\right) \log N_{\jmath-1}-\log P\left(N_{\jmath-1}\right) .
\end{aligned}
$$

Since $\log N_{\jmath}>j \log N_{\jmath-1}$ we have

$$
\frac{\log N_{2}+\cdots+\log N_{\jmath-2}}{\log N_{\jmath-1}}<\frac{2}{\jmath-1} .
$$

We may assume that $N_{j}^{\varepsilon} P\left(N_{j}\right)>1$ and $N_{j}^{-\varepsilon} P\left(N_{j}\right)<1$ for all $\jmath$. Then $N_{\jmath}^{-\varepsilon}<P\left(N_{\jmath}\right)$ $<N_{\jmath}^{\varepsilon}$ and hence

$$
\left|\frac{\log P\left(N_{j}\right)}{\log N_{j}}\right|<\varepsilon
$$

Hence we have

$$
\begin{aligned}
\left|\frac{\log P\left(N_{2}\right)+\cdots+\log P\left(N_{\jmath-2}\right)}{\log N_{\jmath-1}}\right| & \leqq \varepsilon \frac{\left(\log N_{2}+\cdots+\log N_{\jmath-2}\right)}{\log N_{\jmath-1}} \\
& <\frac{2 \varepsilon}{j-1} .
\end{aligned}
$$

Thus the principal term of the numerator is $\left(1+\gamma_{0}\right) \log N_{\jmath-1}-\log P\left(N_{\jmath-1}\right)>$ $\left(1+\gamma_{0}-\varepsilon\right) \log N_{\jmath-1}$, and that of denominator is $\alpha_{0} \log N_{\jmath-1}$. Hence we get

$$
\operatorname{dim}_{H}\left(\bigcap_{\jmath=1}^{\infty} E_{\jmath}\right) \geqq \frac{1+\gamma_{0}-\varepsilon}{\alpha_{0}} .
$$

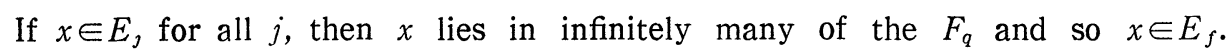
Therefore

$$
\operatorname{dim}_{H}\left(E_{f}\right) \geqq \operatorname{dim}_{H}\left(\bigcap_{\jmath=1}^{\infty} E_{\jmath}\right) \geqq \frac{1+\gamma_{0}-\varepsilon}{\alpha_{0}}
$$

Since $\varepsilon>0$ is arbitrary, we have $\operatorname{dim}_{H}\left(E_{f}\right) \geqq\left(1+\gamma_{0}\right) / \alpha_{0}$.

Finally we consider the case $\alpha_{0} \leqq 1+\gamma_{0}$. It is clear that

$$
E_{f}^{1+r_{0}+\varepsilon} \subset E_{f}^{\alpha_{0}}
$$

for any positive number $\varepsilon$. Then we have from our preceeding results

$$
\frac{1+\gamma_{0}}{1+\gamma_{0}+\varepsilon} \leqq \operatorname{dim}_{H} E_{f}^{\alpha_{0}} \leqq 1
$$

for any $\varepsilon>0$. Hence we get

$$
\operatorname{dim}_{H} E_{f}^{\alpha_{0}}=1
$$

We now show a lemma to obtain the Theorem B-F from Proposition 1.2. 
LEMMA 2.5. The critical exponent $\nu_{0}$ of

$$
\sum_{q \in \mathcal{L}} \frac{1}{q^{\nu}}
$$

is equal to $\gamma_{0}=\sup \left\{\gamma: \overline{\lim }_{N \rightarrow \infty} C_{\mathcal{L}}(N) / N^{r}>0\right\}$, where $C_{\mathcal{L}}(N)=\#\{q \leqq N: q \in \mathcal{L}\}$.

Proof. Let $\nu<\gamma_{0}$. We can choose a sequence $\left\{N_{i}\right\}$ with $N_{j} \rightarrow \infty(i \rightarrow \infty)$ such that $C_{\mathcal{L}}\left(N_{\imath}\right)>2 N_{\imath}^{\nu}$. We also choose a subsequence $\left\{L_{j}\right\}$ of $\left\{N_{i}\right\}$ such that

Then we have

$$
L_{\jmath}>C_{\mathcal{L}}\left(L_{\jmath-1}\right)^{1 / \nu} L_{\jmath-1} \text {. }
$$

$$
C_{\mathcal{L}}\left(L_{j}\right)-C_{\mathcal{L}}\left(L_{\jmath-1}\right)>L_{\jmath}^{\nu} .
$$

We set $\mathcal{L}_{i}=\left\{q \in \mathcal{L}: L_{\jmath-1}<q \leqq L_{j}\right\}$. Then

$$
\sum_{q \in \mathcal{L}} \frac{1}{q^{\nu}}=\sum_{j=1}^{\infty} \sum_{q \in \mathcal{L}} \frac{1}{q^{\nu}}>\sum_{j=1}^{\infty} \frac{C_{\mathcal{L}}\left(L_{j}\right)-C_{\mathcal{L}}\left(L_{j-1}\right)}{L_{\jmath}^{\nu}}>\sum_{j=1}^{\infty} 1=\infty .
$$

When $\nu>\gamma_{0}$, we can show that the series $\sum_{q \in \mathcal{L}} 1 / q^{\nu}$ is convergent by the same way used in the proof of the inequality $\operatorname{dim}_{H} E_{f} \leqq\left(1+\gamma_{0}\right) / \alpha_{0}$.

\section{Proof of Theorem 1.1}

In this section, we prove the Theorem 1.1. Let $g$ be a nonnegative function defined on the set of all natural numbers. Let

$$
E_{g}=\left\{x \in[0,1]:\left|x-\frac{p}{q}\right|<g(q) \text { for infinitely many } q\right\} .
$$

Set

$$
\gamma(\alpha)=\sup \left\{\gamma: \varlimsup_{N \rightarrow \infty} \frac{C_{\alpha}(N)}{N^{r}}>0\right\}
$$

for $\alpha \geqq 1$, where $C_{\alpha}(N)$ is the cardinality of the set $\left\{q \leqq N: g(q) \geqq 1 / q^{\alpha}\right\}$. We define a function $\delta(\alpha)$ as follows

$$
\delta(\alpha)=\left\{\begin{array}{cl}
\frac{1+\gamma(\alpha)}{\alpha} & \text { if } \lim _{N \rightarrow \infty} C_{\alpha}(N)=\infty \\
0 & \text { otherwise. }
\end{array}\right.
$$

Then we have

THEOREM 1.1.

$$
\operatorname{dim}_{H} E_{g}=\min \left\{\sup _{\alpha \geq 1} \delta(\alpha), 1\right\}
$$


Proof. Set

$E=\left\{x \in[0,1]:\left|x-\frac{p}{q}\right|<g(q)\right.$ for infinitely many $\left.q\right\}$,

$E_{\alpha}=\left\{x \in[0,1]:\left|x-\frac{p}{q}\right|<g(q)\right.$ and $g(q) \geqq \frac{1}{q^{\alpha}}$ for infinitely many $\left.q\right\}$

and

$F_{\alpha}=\left\{x \in[0,1]:\left|x-\frac{p}{q}\right|<g(q)\right.$ and $g(q)<\frac{1}{q^{\alpha}} \quad$ for infinitely many $\left.q\right\}$.

Set $F_{\infty}=\bigcap_{\alpha \geq 1} F_{\alpha}$ then it is clear that

$$
E-\bigcup_{\alpha \geqq 1} E_{\alpha} \subset F_{\infty} .
$$

We can show that $\operatorname{dim}_{H} F_{\infty}=0$ as follows. Let

$$
G_{\alpha}=\left\{x \in[0,1]:\left|x-\frac{p}{q}\right|<\frac{1}{q^{\alpha}} \text { for infinitely many } q\right\} .
$$

Then we have

$$
F_{\infty} \subset F_{\alpha} \subset G_{\alpha}
$$

for $\alpha \geqq 1$. Hence $\operatorname{dim}_{H} F_{\infty} \leqq \operatorname{dim} G_{\alpha}=2 / \alpha$. Since $\alpha$ is arbitrary large, $\operatorname{dim}_{H} F_{\infty}=0$.

Therefore $\operatorname{dim}_{H}\left(E-\bigcup_{\alpha \geqq 1} E_{\alpha}\right)=0$ and hence $\operatorname{dim}_{H} E=\operatorname{dim} \bigcup_{\alpha \geqq 1} E_{\alpha}$.

First we show the inequality $\operatorname{dim}_{H} E \geqq \min \left\{\sup _{\alpha \geqq 1} \delta(\alpha), 1\right\}$. Set

$$
H_{\alpha}=\left\{x \in[0,1]:\left|x-\frac{p}{q}\right|<\frac{\chi_{A_{\alpha}}(q)}{q^{\alpha}} \text { for infinitely many } q\right\},
$$

where $A_{\alpha}=\left\{q \in N: g(q) \geqq 1 / q^{\alpha}\right\}$ and $\chi_{A_{\alpha}}$ is the characteristic function of $A_{\alpha}$. Then by the Proposition 1.2, we have

$$
\operatorname{dim}_{H} H_{\alpha}=\min \left\{\frac{1+\gamma(\alpha)}{\alpha}, 1\right\}
$$

if $A_{\alpha}$ is an infinite set and $\operatorname{dim}_{H} H_{\alpha}=0$ if $A_{\alpha}$ finite. Hence $\operatorname{dim}_{H} H_{\alpha}=\min \{\delta(\alpha), 1\}$. Since $E_{\alpha} \supset H_{\alpha}$,

Therefore

$$
\operatorname{dim}_{H} E_{\alpha} \geqq \operatorname{dim}_{H} H_{\alpha}=\min \{\delta(\alpha), 1\} .
$$

$$
\begin{aligned}
\operatorname{dim}_{H} E=\operatorname{dim}_{H} \bigcup_{\alpha \geqq 1} E_{\alpha} & \geqq \sup _{\alpha \geq 1} \operatorname{dim}_{H} E_{a} \\
& \geqq \sup _{\alpha \geqq 1} \min \{\delta(\alpha), 1\} \\
& =\min \left\{\sup _{\alpha \geqq 1} \delta(\alpha), 1\right\} .
\end{aligned}
$$

Next, we show the converse inequality $\operatorname{dim}_{H} E \leqq \min \left\{\sup _{\alpha \geqq 1} \delta(\alpha), 1\right\}$. For positive real numbers $\alpha$, $\beta$ such that $\alpha>\beta$, we set 
$E_{\alpha}^{\beta}=\left\{x \in[0,1]:\left|x-\frac{p}{q}\right|<g(q)\right.$ and $\frac{1}{q^{\beta}}>g(q)>\frac{1}{q^{\alpha}}$ for infinitely many $\left.q\right\}$ and

$$
H_{\alpha}^{\beta}=\left\{x \in[0,1]:\left|x-\frac{p}{q}\right|<\frac{\chi_{A_{\alpha}}(q)}{q^{\beta}} \text { for infinitely many } q\right\} .
$$

It is clear that $E_{\alpha}^{\beta} \subset H_{\alpha}^{\beta}$. So we have, by using Proposition 1.2 again,

$$
\begin{aligned}
\operatorname{dim}_{H} E_{\alpha}^{\beta} & \leqq \operatorname{dim}_{H} H_{\alpha}^{\beta} \\
& =\min \left\{\frac{1+\gamma(\alpha)}{\beta}, 1\right\} \\
& =\min \left\{\frac{1+\gamma(\alpha)}{\alpha} \cdot \frac{\alpha}{\beta}, 1\right\},
\end{aligned}
$$

if $A_{\alpha}$ is infinite and $\operatorname{dim}_{H} E_{\alpha}^{\beta}=0$ if $A_{\alpha}$ finite. Hence

$$
\operatorname{dim}_{H} E_{\alpha}^{\beta} \leqq \min \left\{\delta(\alpha) \cdot \frac{\alpha}{\beta}, 1\right\} .
$$

Fix an $\varepsilon>0$ arbitrary. Define the sequence $\left\{\alpha_{n}\right\}_{n=0}^{\infty}$ by setting $\alpha=1+n \varepsilon$. Then it is easily seen that

$$
\bigcup_{\alpha \geqq 1} E_{\alpha}=\left(\bigcup_{n=1}^{\infty} E_{\alpha_{n}}^{\alpha_{n-1}}\right) \cup E_{1} .
$$

If $E_{1}$ is not empty, then the set $\{q: g(q) \geqq 1 / q\}$ is infinite. Hence $\delta(1)=$ $\left(1+\gamma(1) / 1 \geqq 1\right.$. Therefore $\min \left\{\sup _{\alpha \geqq 1} \delta(\alpha), 1\right\}=1$ and hence the inequality is obvious. So we may assume that $E_{1}$ is empty. For a countable family of sets $\left\{X_{n}\right\}_{n=1}^{\infty}$, the Hausdorff dimension has the following property:

$$
\operatorname{dim}_{H} \bigcup_{n=1}^{\infty} X_{n}=\sup _{n \geq 1} \operatorname{dim}_{H} X_{n} .
$$

So we have

$$
\begin{aligned}
\operatorname{dim}_{H} E & =\operatorname{dim}_{H} \bigcup_{\alpha \geq 1} E_{\alpha} \\
& =\operatorname{dim}_{H} \bigcup_{n=1}^{\infty} E_{\alpha_{n}}^{\alpha_{n-1}} \\
& =\sup _{n \geq 1} \operatorname{dim}_{H} E_{\alpha_{n}}^{\alpha_{n-1}} \\
& \leqq \sup _{n \geq 1} \min \left\{\delta\left(\alpha_{n}\right) \cdot \frac{\alpha_{n}}{\alpha_{n-1}}, 1\right\} \\
& =\min \left\{\sup _{n \geq 1} \delta(1+n \varepsilon) \cdot \frac{1+n \varepsilon}{1+n \varepsilon-\varepsilon}, 1\right\} \\
& \leqq \min \left\{\sup _{\alpha \geq 1} \delta(\alpha) \cdot \frac{\alpha}{\alpha-\varepsilon}, 1\right\}
\end{aligned}
$$




$$
\leqq \min \left\{\frac{1}{1-\varepsilon} \sup _{\alpha \geqq 1} \delta(\alpha), 1\right\} .
$$

Since $\varepsilon>0$ is arbitrary, we have the desired conclusion.

\section{Examples}

For a function $g(q)$ defined on $\boldsymbol{N}$, we set

Then we see

$$
a_{q}=\left\{\begin{array}{cc}
-\frac{\log g(q)}{\log q} & \text { if } g(q)>0 \\
\infty & \text { if } g(q) \leqq 0 .
\end{array}\right.
$$

$$
\left\{q \in \boldsymbol{N}: a_{q}<\alpha\right\}=\left\{q \in \boldsymbol{N}: g(q)>\frac{1}{q^{\alpha}}\right\}
$$

for $\alpha \in \boldsymbol{R}$. Hence the Hausdorff dimension of $E$ is determined by the distribution of the sequence $\left\{a_{q}\right\}$.

PROPOSITION 4.1. If the cardinality of the set $\left\{q \in N: a_{q}<\alpha\right\}$ is finite for any $\alpha \in \boldsymbol{R}$, then $\operatorname{dim}_{H} E=0$.

Proof. By the assumption we have $\lim _{N \rightarrow \infty} C_{\alpha}(N)=\#\left\{q \in \boldsymbol{N}: a_{q}<\alpha\right\}<\infty$. Hence from Theorem 4.1, $\operatorname{dim}_{H} E=0$.

We may apply Proposition 4.1 for following functions

$$
\begin{aligned}
& g(q)=\frac{1}{q^{q}}, \frac{1}{(\log q)^{q}}, \frac{1}{q^{\log q}}, \frac{1}{(\log q)^{\log q}}, \text { etc. } \\
& g(q)=\frac{1}{q^{\varphi(q)}}
\end{aligned}
$$

where $\varphi(q)$ is the Euler function.

$$
g(q)=\frac{1}{a^{q}},
$$

where $a$ is a constant with $a>1$.

$$
g(q)=\frac{1}{q !} .
$$

By Theorem 1.1, we have

PROPOSITION 4.2. If the sequence $\left\{a_{q}\right\}$ is distributed in an interval $[s, t]$ $(2 \leqq s \leqq t)$ in such a way that the limit 


$$
\lim _{N \rightarrow \infty} \frac{\#\left\{q \leqq N: a_{q} \in[s, \alpha]\right\}}{N^{r}}
$$

exists and its value is positive for any $s<\alpha$, where $\gamma$ is a constant $0<\gamma \leqq 1$, then

$$
\operatorname{dim}_{H} E=\frac{1+\gamma}{s} .
$$

We can apply Proposition 4.2 for following cases:

(1) $\left\{a_{q}\right\}$ is uniformly distributed in $[s, t]$. Then $\gamma=1$ and $\operatorname{dim}_{H} E=2 / s$.

(2) $a_{q}=s+|\sin q|$.

The sequence $\{q / \pi\}$ is uniformely distributed in $[0,1] \bmod 1$. Hence $\left\{a_{q}\right\}$ satisfies the condition of Proposition 4.2 for $\gamma=1$ and $\operatorname{dim}_{H} E=2 / s$.

Example 4.3. We set $a_{q}=s+k$ if $q \equiv k(\bmod n)$, where $n$ is a fixed natural number and $s(\geqq 2)$ is also fixed. Then if $s+k<\alpha \leqq s+k+1$,

$$
\begin{aligned}
C_{\alpha}(N) & =\#\left\{q \leqq N: \frac{1}{q^{a_{q}}}>\frac{1}{q^{\alpha}}\right\} \\
& =\#\{q \leqq N: q \equiv 0,1, \cdots, k-1(\bmod n)\} \\
& \sim \frac{k}{n} N .
\end{aligned}
$$

Hence $\gamma(\alpha)=1$ and we have

$$
\operatorname{dim}_{H} E=\sup _{s<\alpha} \frac{2}{\alpha}=\frac{2}{s} .
$$

\section{REFERENCES}

[1] I. Borosh AND A.S. Fraenkel, A generalization of Jarník's theorem on Diophantine approximations, Indag. Math., 34 (1972), 193-201.

[2] K.J. Falconer, Fractal Geometry, John Wiley and Sons, 1990.

[3] K. J. FAlconer, The Geometry of Fractal Sets, Cambridge University Press, 1985.

Department of Mathematics

COLLEGE OF SCIENCE

UNIVERSITY OF THE RYUKYUS

NishiHaRA, OKINAWA 903-01

JAPAN 